\title{
IN SILICO DESIGN OF BENOXAZOLE BEARING AZETIDINONE DERIVATIVES AS VEGFR-2 AGONIST IN CANCER
}

\author{
LEYANA PN*, MANJU PT, MEENU VIJAYAN
}

Department of Pharmaceutical Chemistry, College of Pharmaceutical Sciences, Government Medical College, Trivandrum, Kerala, India. Email: leyanapn5@gmail.com

Received: 11 July 2021, Revised and Accepted: 01 October 2021

\section{ABSTRACT}

Objective: Cancer is a group of disease characterized by uncontrolled growth of cells. The objective of the study includes the in silico designing of benzoxazole bearing azetidinone derivatives as Vascular Endothelial Growth Factor 2 in cancer.

Methods: In silico design of proposed derivatives was conducted using tools such as AutoDock Vina, ACD Lab ChemSketch ver. 12.0, Prediction of Activity Spectra for Substances online, molinspiration, and Swiss ADME. The derivatives obeying Lipinski's Rule of Five in accordance with molinspiration were selected for docking studies.

Results: The data obtained from molinspiration revealed that the designed derivatives have physical and chemical properties meant for an orally bioavailable drug. From the docking studies derivatives BT1 and BT5 showed high docking score which indicate that these derivatives possess high affinity and high polar interaction towards protein 4DBN.

Conclusion: The designed benzoxazole bearing azetidinone derivatives were found to possess good binding affinity and good interaction in the binding pocket of the target 4DBN. Therefore, these derivatives are expected to exhibit good anticancer property with minimal side effects.

Keywords: Cancer, Vascular endothelial growth factor 2, Docking, AutoDock vina, Sorafenib.

(C) 2021 The Authors. Published by Innovare Academic Sciences Pvt Ltd. This is an open access article under the CC BY license (http://creativecommons.org/ licenses/by/4.0/) DOI: http://dx.doi.org/10.22159/ajpcr.2021v14i11.42706. Journal homepage: https://innovareacademics.in/journals/index.php/ajpcr

\section{INTRODUCTION}

Cancer is a disease caused by an uncontrolled growth of abnormal cells. It is the second leading cause of death globally. The major types of cancers are sarcoma, melanoma, leukemia, lymphoma, and carcinomas. Tumor cells do not have any programming so that they do not provide any physiological function [1-3].

Benzoxazole and its derivatives constitute an important class of heterocycles in drug discovery. These derivatives show anti-bacterial, anti-fungal, anti-histaminic, and anti-cancer properties [4-6]. Azetidinones are the important scaffold with anti-tubercular, anti-HIV, anti-inflammatory activity etc. [7].

Vascular endothelial growth factor (VEGF) signaling pathway creates a vital role in governing tumor angiogenesis. Inhibition of the signaling pathway is considered as an effective therapeutic target for tumor angiogenesis inhibition and successive tumor growth. VEGF receptor (VEGFR) is a well-known target for many antineoplastic drugs including Sorafenib and Axitinib [8,9].

\section{METHODS}

ACD Lab ChemSketch ver. 12.0

ACD Lab ChemSketch ver. 12.0 is a software program helpful in the drawing and naming of chemical structures of various organic compounds. It gives information about characteristics such as calculation of molecular descriptors which include molecular weight, Molar volume, surface tension, Parachor, Polarizability, and Refractive index.

\section{Molinspiration}

It is a free web tool for the calculation of different molecular properties needed in QSAR, which includes $\log \mathrm{P}$, number of rotatable bonds, number of hydrogen bond acceptors, number of hydrogen bond donors, and number of violations. The oral bioavailability of synthesized derivatives can be predicted by these molecular descriptors which come under Lipinski Rule of Five. Molinspiration also help to predict the bioactivity score for the most important drug targets.

Prediction of activity spectra for substances (PASS) online

It is an online software for the prediction of biological activities. The results can be obtained with a list of over 4000 kinds of pharmacological activities consisting of $\mathrm{Pa}$ and $\mathrm{Pi}$ values arranged in their descending order of differences.

- If Pa $>0.7$, the substance is very likely to exhibit activity and chance of being an analog of a known pharmaceutical agent

- If $0.5<\mathrm{Pa}>0.7$, the substance is likely to exhibit activity and substance is unlike known pharmaceutical agent

- If $\mathrm{Pa}<0.5$, substance unlikely to exhibit activity and a chance of being a new chemical entity.

\section{Swiss ADME}

Swiss ADME is a web tool with free access to the physicochemical, pharmacokinetic, and similar properties of powerful molecules. It produce predictive models using various methods such as BOILED-Egg (Fig. 1), $\log$ P, and bioavailability Radar (Fig. 2). In BOILED-Egg model, the white region denotes high probability of passive absorption by the gastrointestinal tract and yolk region is for high probability of brain penetration. In bioavailability radar, the pink area represents the optimal range for each property (Lipophilicity: XLOGP3 between -0.7 and +5.0 , size: Mol Wt. between 150 and $500 \mathrm{~g} / \mathrm{mol}$, polarity: TPSA between 20 and 130 Ao, solubility: $\log \mathrm{S}$ not higher than 6 etc. The parameters are tabulated in Table 1

\section{Protein data bank}

Protein data bank is a resource for various proteins and macromolecules. Each entry in PDB is represented by a PBD ID, which 
Table 1: Pharmacokinetic study by Swiss ADME of derived derivatives

\begin{tabular}{|c|c|c|c|c|c|}
\hline Compound code & Formula & GI absorption & BBB penetration & P-gp substrate & Pains \\
\hline BT1 & C18H14CIN303S & Yes & No & No & 0 alert \\
\hline BT2 & C18H13Cl2N3O3S & Yes & No & No & 0 alert \\
\hline ВТ3 & C18H14CIN304S & Yes & No & No & 0 alert \\
\hline BT4 & C19H16CIN303S & Yes & No & No & 0 alert \\
\hline BT5 & C18H13CIN405S & Yes & No & No & 0 alert \\
\hline Standard & C20H16ClFN4O3 & Yes & No & No & 0 alert \\
\hline
\end{tabular}

Table 2: Molecular descriptors of derivatives derived using ACD Lab Chemsketch V 12.0

\begin{tabular}{|c|c|c|c|c|c|c|}
\hline Compound code & Parachor $\left(\mathrm{cm}^{3}\right)( \pm 6.0)$ & $\begin{array}{l}\text { Molar volume } \\
\left(\mathrm{cm}^{3}\right)( \pm 5.0)\end{array}$ & $\begin{array}{l}\text { Polarizability } \\
\left(10^{-24}\right)( \pm 5.0)\end{array}$ & $\begin{array}{l}\text { Molar refractivity } \\
( \pm 0.4)\end{array}$ & $\begin{array}{l}\text { Surface tension } \\
\text { (Dyne/cm) }( \pm 5.0)\end{array}$ & $\begin{array}{l}\text { Refractive } \\
\text { index }( \pm 0.03)\end{array}$ \\
\hline BT 1 & 756.1 & 256.4 & 40.03 & 100.98 & 75.6 & 1.717 \\
\hline BT 2 & 793.3 & 267.2 & 41.94 & 105.80 & 77.6 & 1.722 \\
\hline BT 3 & 771.3 & 253.2 & 40.63 & 102.51 & 86.0 & 1.743 \\
\hline BT 4 & 794.4 & 272.1 & 41.86 & 105.51 & 72.6 & 1.703 \\
\hline BT 5 & 813.2 & 267.4 & 42.42 & 107.01 & 85.4 & 1.732 \\
\hline
\end{tabular}

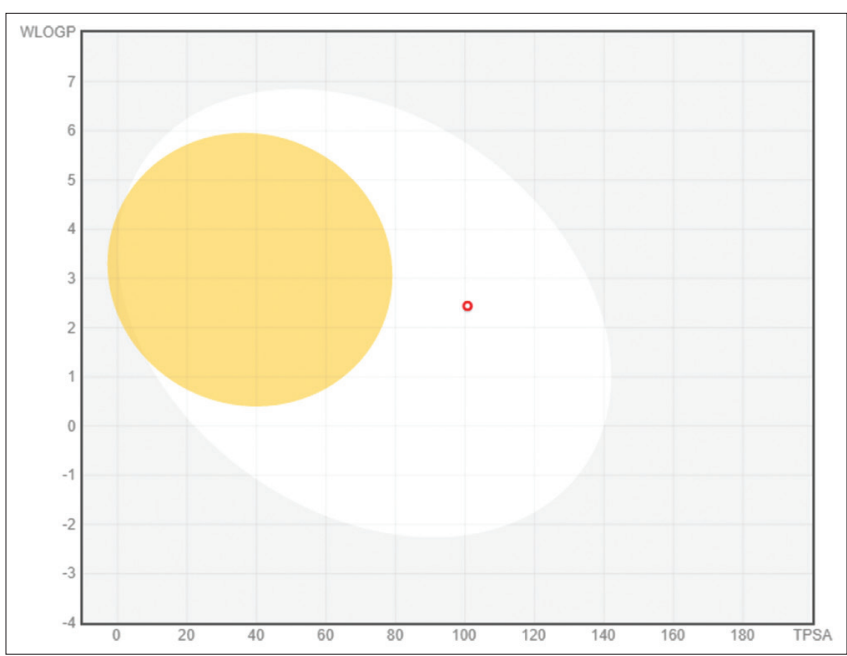

Fig. 1: Boiled egg model of BT1

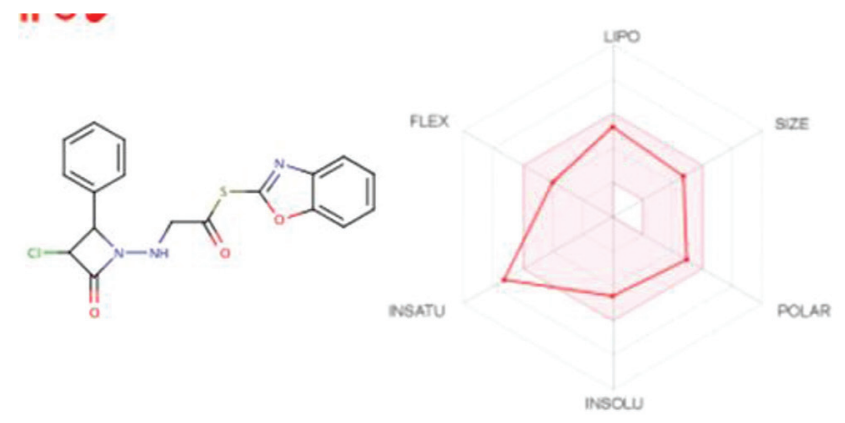

Fig. 2: Bioavailability radar diagram of BT1

is a four-character unique identifier called PDB ID, for example, 4DBN. Fig. 3 (Crystal Structure of the Kinase domain of Human B-raf with a $[1,3]$ thiazolo [5,4-b] pyridine derivative).

\section{Molecular docking}

Molecular docking studies are the computational techniques used to determine the interaction of two molecules and to find out the best orientation of ligand which would form a complex with the intended receptor. PyRx and PyMol are the two programs which are used for this purpose. PyMol is generally adopted for protein preparation and their

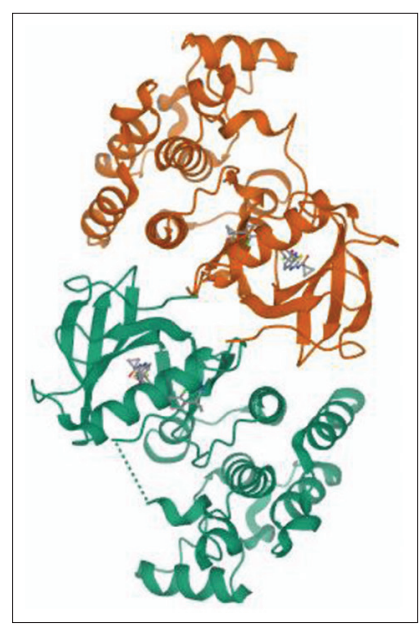

Fig. 3: Structure of vascular endothelial growth factor receptor 2 (PDB ID- 4DBN)

visualization whereas PyRx provide a better platform for docking of ligand with receptor [10].

\section{Protein preparation}

Protein preparation can be done by using Pymol where the protein is obtained from Protein Data Bank. This protein structure can be cleaned by various commands like remove $<>$ resn $<>\mathrm{HOH}$ (for removing water) remove $<>$ resn $<>$ DSN (for detergents), remove $<>$ resn $<>$ IRE and also small molecules to be eliminated. Finally hydrogen atom should be added to the protein structure.

\section{Ligand preparation}

The 2D chemical structure of ligands was drawn using ACD Lab Chemsketch ver. 12.0 and generated smiles notation. This smiles notation is being converted into 3D PDB format with the help of freely accessible Corina Online Software.

\section{Docking by autodock vina}

Docking was performed using PyRx software program. Selected derivatives were loaded into navigation platform. The cleaned protein was converted into macromolecules. The docking procedure was done by clicking Vina wizard start button and adjusting the grid size. The docking scores were obtained which are shown in the Table 2. 
Table 3: Lipinski's Rule analysis of proposed benzoxazole substituted azetidinone derivatives using molinspiration analysis

\begin{tabular}{|c|c|c|c|c|c|c|c|}
\hline Compound code & Structure & Mol. Wt (g/mol) & HA & HD & $\log P$ & nrotb & $\mathrm{n}$ violations \\
\hline BT1 & & 387.84 & 6 & 1 & 3.48 & 6 & 0 \\
\hline ВT2 & & 422.28 & 6 & 1 & 4.15 & 6 & 0 \\
\hline ВT3 & & 403.83 & 2 & 7 & 3.42 & 6 & 0 \\
\hline BT4 & & 401.86 & 1 & 6 & 3.92 & 6 & 0 \\
\hline BT5 & & 432.83 & 1 & 9 & 3.42 & 7 & 0 \\
\hline Standard (sorafenib) & & 414.81 & 3 & 7 & 4.03 & 5 & 0 \\
\hline
\end{tabular}

Mol.wt: Molecular weight, HA: Hydrogen bond acceptors, HD: Hydrogen bond donors, nrotb: Number of rotatable bonds

Table 4: Prediction of biological activity of derivatives by PASS

\begin{tabular}{llll}
\hline Compound code & Activity & Pa & Pi \\
\hline BT 1 & Anti-cancer & 0.829 & 0.009 \\
BT 2 & Anti-cancer & 0.780 & 0.014 \\
BT 3 & Anti-cancer & 0.811 & 0.010 \\
BT 4 & Anti-cancer & 0.797 & 0.012 \\
BT 5 & Anti-cancer & 0.788 & 0.013 \\
\hline
\end{tabular}

PASS: Prediction of Activity Spectra for Substances

Table 5: Docking score of derivatives and standard (sorafenib) with protein 4DBN (ligand binding domain of vascular endothelial growth factor 2)

\begin{tabular}{lll}
\hline S. No. & Compound code & Docking score $(\mathrm{Kcal} / \mathbf{m o l})$ \\
\hline 1. & BT1 & -8.6 \\
2. & BT2 & -8.4 \\
3. & BT3 & -8.1 \\
4. & BT4 & -8.0 \\
5. & BT5 & -9.3 \\
6. & Standard (Sorafenib) & -10.1 \\
\hline
\end{tabular}

Visualization and analysis

The hydrogen bond, hydrophobic bond, and pi-pi interactions were analyzed using PyMol molecular graphic system. PyMOL can be used to develop a well-defined 3D image of small molecules, biological macromolecules such as proteins [11].

\section{RESULTS}

Molecular descriptors

The evaluation of molecular descriptors was done using ACD Lab Chemsketch ver. 12.0 is shown in Table 2.

\section{Molinspiration}

Molinspiration analysis is used to calculate the physicochemical parameters and to analyze Lipinski's Rule of Five. The results are shown in Table 3.

\section{PASS online software}

In this study, PASS online was performed. The derivatives showed good anti-cancer properties which is tabulated in Table 4 .

\section{Prediction of ADME properties}

Pharmacokinetic properties evaluated through Swiss ADME, the data were obtained are tabulated in Table 1.

\section{Molecular docking}

The docking analysis was performed through PyMol and PyRx software programs and the results are shown in Table 5 and the figures are shown in Fig. 4. 


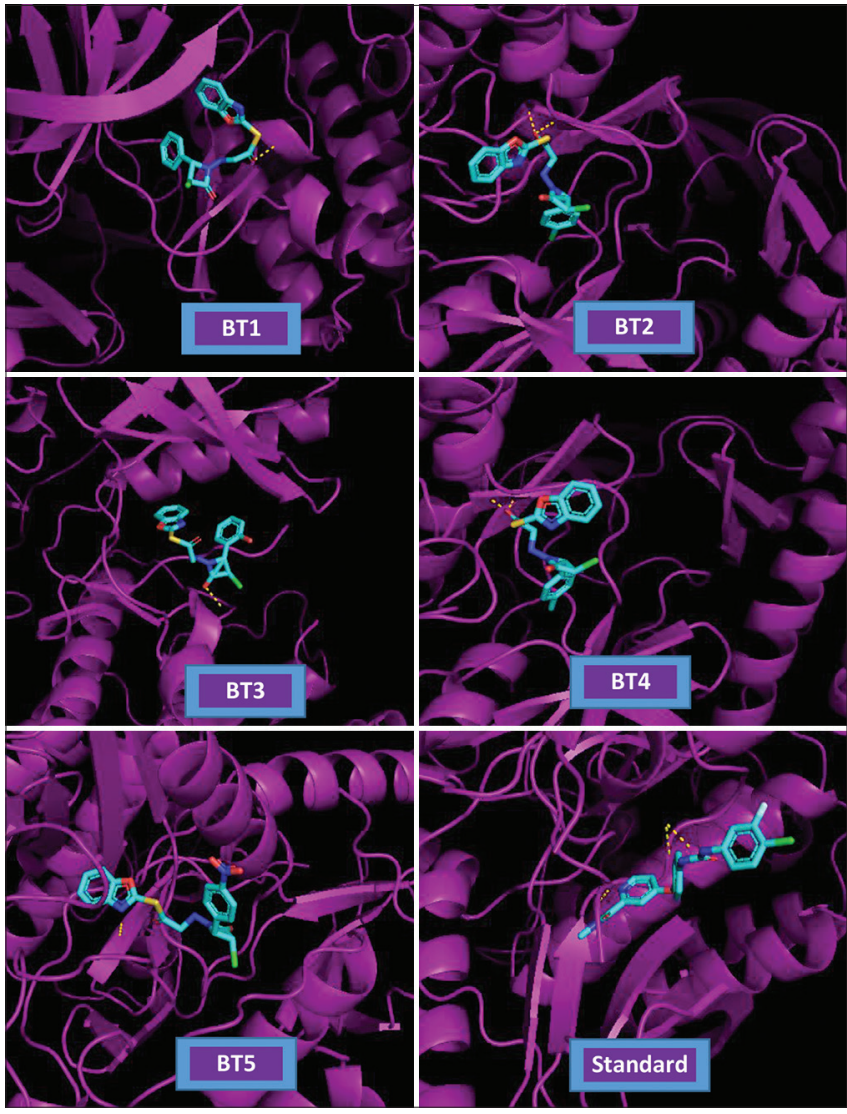

Fig. 4: Docked images of derivatives and standard (sorafenib) with protein 4 DBN (ligand-binding domain of vascular endothelial growth factor 2. Purple color wires - Structural alignment of protein VEGFR-2 (4DBN); Yellow color dotted lines Various amino acid interactions

\section{DISCUSSION}

Using ACD Lab ChemSketch ver. 12.0, we have drawn five benzoxazole substituted azetidinone derivatives and found their molecular properties such as parachor, molar refractivity, surface tension, molar volume, polarizability, and refractive index. All these properties reveal a relationship between structural properties of chemical compounds and the biological activities. The Lipinski rule and its violation are verified in molinspiration cheminformatics as mentioned in Table 3. Lipinski rule states that the molecular weight should not exceed $500 \mathrm{Da}$, not more than 10 hydrogen bond acceptors and 5 hydrogen bond donors. The compounds that violated this rule are eliminated from further proceedings as they are unfit for docking studies. The Lipinski rule plays major role in pharmacokinetic properties like absorption, distribution, metabolism and elimination. The rule of five and drug likeness score of these five derivatives were found to possess good physio-chemical properties and is expected to be orally bioavailable. The biological activity of derivatives was predicted by PASS online software and benzoxazole bearing azetidinone derivatives were found to possess good anticancer activity (Pa more than 0.7 ) as shown in Table 1 . The pharmacokinetic properties were predicted through Swiss ADME software. It showed that all the derivatives exhibit high gastro intestinal absorption and are non BBB premiant together with zero alert for PAINS. The molecular docking analysis of the selected derivatives with the receptor VEGFR-2 (4DBN) was evaluated. Schematic 3D representation of derivatives with receptor VEGFR-2 (4DBN) was obtained and shown in Fig. 4. The docking score of derivatives and the standard (sorafenib) with 4DBN is given in Table 5. Various hydrogen bond interactions were shown with Ser 535 for derivative BT1, Val 599 for derivative BT2, Phe 594 for derivative BT3, Asp 593 for derivative BT4, and Phe 582 for derivative BT5. Hence, all these five derivatives are expected to have good in vivo and in vitro anti-cancer activity.

\section{CONCLUSION}

The present study scientifically revealed the in silico design, ADME prediction, and docking studies to predict anticancer activity. We have selected five benzoxazole substituted azetidinone derivatives and all the compounds showed good molecular properties. Based on the analysis of Lipinski rule of five, all the derivatives passed the rule of five and therefor these compounds were further preceded to pharmacokinetic and docking studies. PASS online predicted anti-cancer activity for those derivatives. Swiss ADME studies resulted with all the five derivatives to be orally bioavailable and they do not cross BBB. From docking scores, we can conclude that the designed benzoxazole substituted azetidinone derivatives are found to have good interaction in binding pocket of target 4DBN, derivatives possess good anticancer activity with high binding affinity. So these compounds are expected to possess good anticancer property with minimal side effects.

\section{ACKNOWLEDGMENT}

This study was supported by College of Pharmaceutical Sciences, Government Medical College, Trivandrum.

\section{AUTHORS' CONTRIBUTIONS}

The $1^{\text {st }}$ and $2^{\text {nd }}$ author contributed to the entire work and drafted the manuscript and the $3^{\text {rd }}$ author participated in docking studies.

\section{CONFLICTS OF INTEREST}

The authors confirm that this article content has no conflicts of interest.

\section{REFERENCES}

1. Harshitha T, Vinay Kumar T, Vineetha T. In silico characterization, molecular docking, and in vitro evaluation of triazole derivatives as potential anticancer agents. Asian J Pharm Clin Res 2021;14:22-8.

2. Pappachen LK, Zachariah Sm, Chandran D. In silico design, synthesis and charecterization of some novel benzothiazole derivatives as anticancer agents. Asian J Pharm Clin Res 2017;10:150-5.

3. Sawant S, Shegokar R. Cancer research therapy, where we today. Int J Cancer Ther 2014;2:1-5.

4. Kakkar S, Lim SM. Benzoxazole derivatives: Design, synthesis and biological evaluation. Chem Central J 2018;12:1-16.

5. Laeeq S, Sirbaiya AK. Benzoxazole: Progress report on chemistry, synthesis and biological activities. Indo Am J Pharm Res 2013;3:2-5.

6. Jadhav RR, Srikanth G. Synthesis of some benoxazole derivatives and their antimicrobial activity. J Pharm Res 2011;4:3562-5.

7. Rockade Y, Dongre N. Azetidinone (beta lactam) derivatives: An emerging antimicrobials. Asian J Microbiol Biotech Environ Sci 2009;11:109-14.

8. Modi SJ, Kulkarni VM. Vascular endothelial growth factor receptor (VEGFR-2) inhibitor; medicinal chemistry perspective. Med Drug Discov 2019;2:100009.

9. Ei-Helby AG, Sakr H. Design, synthesis, molecular docking and anticancer activity of benzoxazole derivatives as VEGFR-2 inhibitors. Arch Pharm 2019;352:1900113.

10. Rentzsch R, Renard BY. Docking small peptides remains a great challenge: An assessment using AutoDock Vina. Brief Bioinform 2015;16:1045-56

11. Rauf MA, Zubair S. Ligand docking and binding site analysis with pymol and Autodock Vina. Int J Basic Appl Sci 2015;4:168-77. 